\title{
Precursors to using Energy data as a Manufacturing Process Variable
}

\author{
Neil Brown, Rick Greenough, Konstantin Vikhorev \\ Institute of Energy and Sustainable Development, DeMontfort University, \\ Leicester, UK.
}

\begin{abstract}
Energy efficiency can often learn much from manufacturing in terms of available analysis techniques, from basic time series analysis through to fuzzy and knowledge based systems and artificial intelligence. On the other hand, manufacturing in many sectors has yet to make use of energy data much beyond finance. Techniques such as complex event processing and data stream analysis can be applied in near real time to determine process health. Conventional energy data, with a half-hourly time interval through fiscal metering, has been sufficient for off-line process control in the past, but to increase the utility of manufacturing energy data, a step change is needed in data frequency, accuracy, precision, portability, and documentation. This paper brings together co-dependent issues of data structure, data quality, and front-end instrumentation which advanced processing techniques must build on, discussing what must be done to use gather and use energy data more effectively, to reduce energy use and emissions, improve quality, and save costs.
\end{abstract}

Keywords: Energy, efficiency, data, manufacturing, standards

\section{Introduction}

In the UK, $44 \%$ of energy is used by activities within buildings [1], and around $54 \%$ of this is from manufacture. Generally speaking, specific energy use for many modern production methods can drop [2], which bodes well for pressing needs to meet greenhouse gas emission targets, meet any generating shortfall, and to save industrial energy costs. Advanced metering is a useful way to keep track of energy use, gathering building energy data remotely (typically half-hourly), and is increasing in use [3]. While mainly currently used for billing purposes, it offers useful datasets to study energy use [4-5], and ultimately massively streamlined ways to find energy savings [6-7]: Building and manufacturing control faults can be identified $[5,8]$ such as those causing building services to run unnecessarily (e.g. over-cooling, unoccupied heating), in some cases up to $30 \%$ of an estate shows unoccupied (night or weekend) heating [4-5], compressed air production is also a common example of detectable plant running outside unoccupied times. Electrical baseloads can be identified, and reduced by up to $20 \%$ [9], sometimes using quite low-tech interventions such as switch-off campaigns. Meta analyses enable us to gauge the energy use of entire commercial sectors [10]. In general, analysis of these data offers a potentially massive benefit to building users, designers, utilities and policymakers in understanding building energy wastage $[5,7$, 11-14]. There are numerous uses of high frequency energy data, yet common problems include compatibility between datasets, data quality, and completeness [15]. It becomes clear that improving compatibility of energy data is synonymous with improving its usefulness. High level energy management standards are becoming well documented, showing procedures e.g. [15-16] and performance metrics, and energy efficiency prognosis [17]. However, format conversion still takes much time, and inconsistencies in documentation, and data quality cause major reliability issues [5]: When analysing meta datasets, inconsistencies become clear even in notation methods for such fields as addresses [4], or metering point numbers, such that results (and even their input to government policies) are skewed by e.g. double counting. Also, critical data can be ignored, because inconsistent formatting often means that a database server is not capable of processing it. Data quality issues arise for many reasons, the most common being transmission inconsistencies where data contains gaps, or artifacts [4]. This becomes more common in factories where EMI can be high. As new energy data analysis techniques emerge [12], automated processing benefits from consistent data with automated error detection [7, 12-13]. Data structure becomes crucial, notably an understanding of how measurements may be grouped together. For example, electrical power is easily measured for a single machine [18] but gas use may only be available for a whole building.

Gathering enough energy data to control production has been historically difficult and costly, but instrumentation costs have dropped in recent years. Key to the improved ease of use (and deployment) of sensing systems is the idea of noninvasive instrumentation [19]. To limit production line downtime, rapid deployment is obviously useful, and this is where non-invasive technologies come in. These include, for example, ultrasonic fluid sensing and clip on current sensing. Improvements in industrial computing make it cost effective to pre-process high frequency sampled data on the fly, with near real time processing of streaming data, i.e. complex event processing [20] and using sensor fusion and fuzzy systems [21]. This means that should an energy fault, or a manufacturing fault be detected from energy data, we can take action well before excessive scrap and (energy) wastage occurs. This clearly offers cost and environmental advantages over off-line methods of energy and production management.

Once energy data documentation is fully understood, applications beyond ordinary energy management become easier. Energy use is of increasing interest outside traditional heavy industry (such as steelmaking), and is an increasing proportion of costs in many areas, e.g. semiconductor manufacture [22]. In many industrial processes, the actual time that machines are running can be relatively short, with time waiting for fresh parts or material to process, or tooling changeovers. This leads to machines running on standby, with 
no useful manufactured output. It is possible to identify modes of energy consumption directly from energy data, which generally apply to the type of appliance examined, such as idle (continuous), idle (intermittent), runtime and cutting [23] for a machine tool, or running, active standby, passive standby and off for a generalized electrical machine [9], and we can ensure low power modes whenever possible. Load shifting becomes possible, to schedule heavy loads against variable rate tariffs, and also companies approaching their supply limit are able to schedule certain tasks to obviate supply upgrade costs [18]. Ultimately, process health is revealed by studying power consumption. Examples include increased electricity use for blunt tooling, or increased power needed to pump fluid through contaminated piping. Figure 1 shows the basic concept of using energy data to reveal process health.

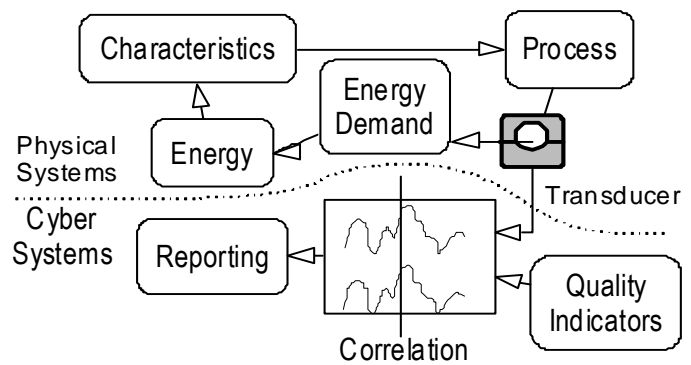

Figure 1: Basic principle of energy data as a manufacturing process variable.

Another form of energy analysis based on the second law of Thermodynamics is Exergy Analysis. As well as offering the usual efficiency gains, Exergy Analysis can also act as a quality control parameter processes that require minimizing temperature gradients within the final product. This offers an extra incentive for deploying online monitoring systems.

\section{DATA STRUCTURES}

The authors have explained [24] that energy data structure issues while apparently complex, fall into four main areas: Temporal (e.g. time and date issues, handling time driven data errors), Accuracy and Precision (including sensing devices and energy data quality), Data documentation (e.g. multipliers for $\mathrm{kWh}$, factory zones), and operational documentation (e.g. known down-time, data transmission issues). Fortunately, some standard approaches exist which can guide us: The International Standard for spectral data exchange [25] gives a good example of a data structure specifically aimed at complex data transmission. We must remember that timestamps are likely to require resolution down to seconds, and we must ensure, or at least be sensitive to compatibility with week numbers. Free running sampled data (e.g. milliseconds) however can be assumed to be processed and logged at longer intervals before storing in energy databases. Crucial at this phase is to specify the accuracy and precision of timestamps, and latency [26]. Following on from this, there may be nontransmission periods a standard reporting method is needed, requiring similar error codes to those described in [27]. Even low-level data transmission can carry equipment status using appropriate keywords [28] and using appropriate codes for phenomena such as power (factor), current and voltage, and fiscal meters may be treated as a credible reference [29], and measuring procedures can be specified to appliance level [30] and an extensible data structure facilitates this and [31] offering appropriate descriptors. An extensible data structure with event reporting capability exists [26, 33-34], and while aimed at supply-side electricity distribution, suggests an appropriate system for energy event reporting, hierarchy descriptors. A new standard structure can build on this useful work.

\section{A. Disaggregation}

Figure 2 represents results of a thought experiment to identify energy feeds in a generalized factory. Levels of disaggregation possible are balanced by available instrumentation as much as need for clear data.

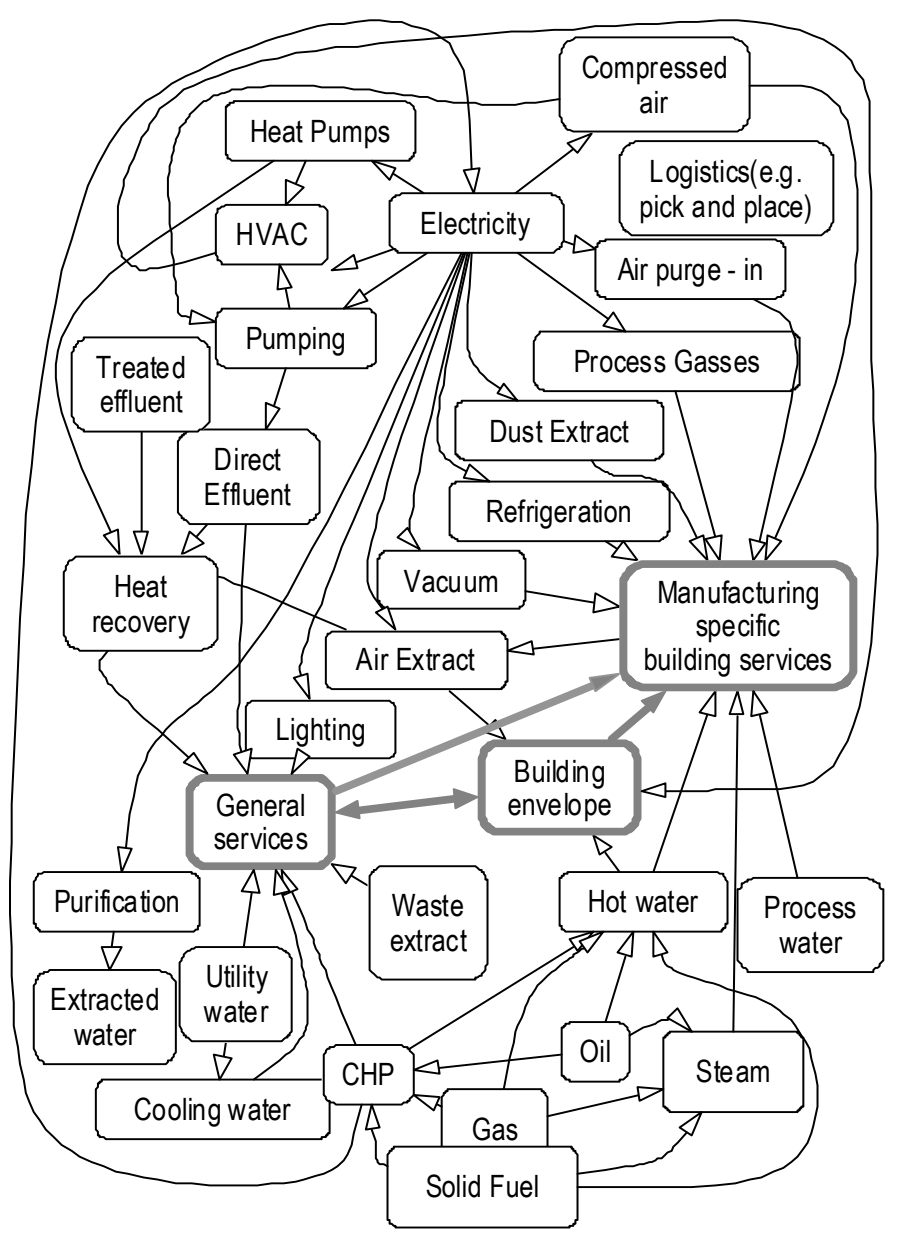

Figure 2. Energy related Factory Building Services

The type of data structure we need in manufacturing energy analysis is closely linked to these issues of disaggregation. While aggregated energy use at sector level is useful for policymakers and utilities [2], and it is clear that a common language of performance indicators is needed between sectors, describing where energy flows around a factory becomes a more interesting challenge. It is clearly harder to manage energy for a process when some services are shared between machines, production lines, and manufacturing zones . Machine level energy feeds can be easily broken down to e.g. 
electrical with possible local hydraulic and vacuum, pneumatic, all with electrical feeds [35] with this caveat, centralized services include, lighting, air conditioning, heating, fume extraction, centralised swarf removal and most usually compressed air. We need to distinguish between building services energy feeds, those for manufacturing related technical building services, and energy directly used by production machines.

\section{ENERGY DATA QUALITY}

Figure 3 shows the entity relationships for a typical energy dataset. When logically presented, we see that the data structure falls into four sections: temporal data, accuracy and precision of data, supporting documentation, and energy data documentation.

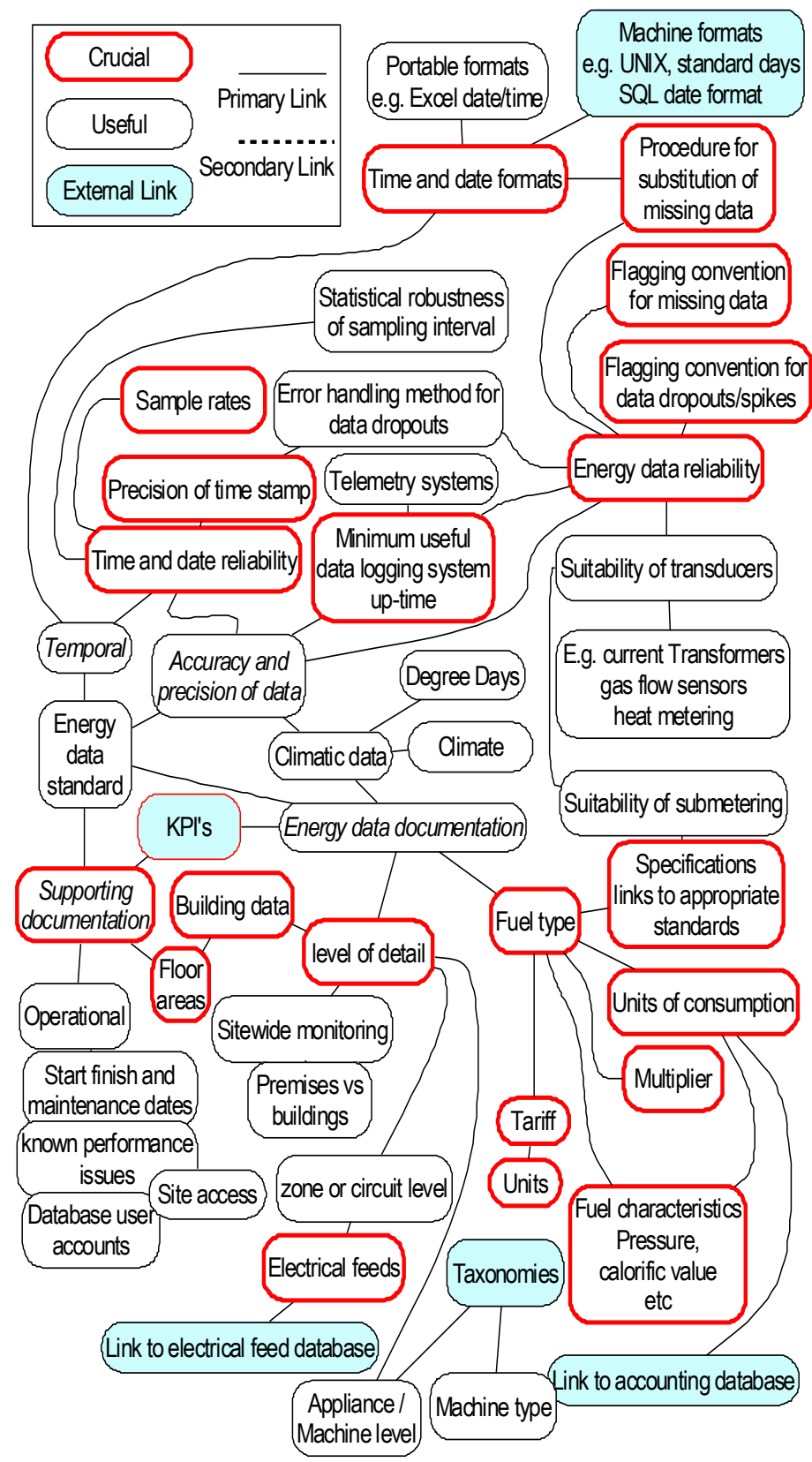

Figure 3. Energy data entities.
The entities from Figure 3 may be distilled thus:

\section{A. Temporal}

Date formats, time stamping and sample rates, are vital to energy data, but often error prone, so commonly, some data may not be analysed. It's common practice to substitute data from similar periods, or interpolation is used with some detail loss [6]. A minimum acceptable data quality level could (should) be stipulated for time stamping in reliability and precision, but also procedures described for handling of data errors, and logging them. Basic telemetry systems reliability should be stipulated, whereby should data dropouts cross a threshold, cleaning is abandoned in favor of corrective action on hardware or software.

\section{B. Data Accuracy and Precision.}

Even for a clean, error free dataset, another shortcoming is that accuracy and precision of time stamping and energy data are rarely quantified. One example is that the basic nature of (low power) electronics in conventional energy data loggers alows some time errors to accumulate. One upshot of this is that half hourly data, for example, may not be recorded on the half-hour, hampering analysis and requiring resampling. Transducer accuracy and precision is also important, for example current transformers (CTs) for electricity monitoring, are unreliable for certain low currents, depending on the units peak load. Climate data is widely used calculation of predicted loads and outside air temperature (and other weather) probe location should be documented. Where transmissions dropouts occur, this may produce, apparent zeros in consumption data, followed often by a very large accumulated spike when transmission resumes. This hampers analysis considerably, but could be circumvented with a flagging convention, to allow skipping over faulty data, or to trigger data cleaning.

\section{Operational Documentation}

This is needed so that energy feed descriptors, and database specific operational data, also performance issues downtime for radio telemetry apparatus are known. Documentation can include user accounts, permissions and privileges for database access, details of access to metered zones, buildings or sites and data gathering hardware/software maintenance schedules.

\section{Data Documentation}

This should be stored within an energy dataset, notably energy and fuel types, (e.g. gas caloric values), and conversion factors to $\mathrm{kWh}$. Any data structure must be open to the inclusion of tariff information, for compatibility with variable rate tariffs and load leveling.

\section{INSTRUMENTATION}

Introducing submetering, and heavy disaggregation of energy feeds in factories has potential to be more complicated than in other commercial buildings, because of costs of production loss, safety issues at time of fitting, and future need for reconfiguring machine layouts as production changes, to replace faulty instruments in future, and reinstalling sensors after machine maintenance. The good news is the on-site 
presence of electricians and engineers in many factories can speed fitting of instrumentation. For permanent submetering, meter pulses are traditionally monitored using a compatible electricity meter (this is also true for gas metering). Electricity meters may give a dry relay contact closure output, meaning that the output is essentially a switch, such that extra circuitry may be needed before the data logger input to electrically 'debounce' the signal. Specifications for pulse outputs are given by BSI [35] with explanations of terms [36]. Where a pulse output meter is fitted (which is rare for disaggregated feeds in manufacturing), adding logging equipment with no further disturbance is straightforward. Current transformers may detect power consumption on either single phase, or multiphase - the proviso being that a clear phase is required for each sensing element (Live Neutral and Earth cable, for example, would not be suitable) Current transformers range from low cost transducers requiring signal conditioning, to 'Clamp Meters' self contained units with signal conditioning, designed for spot measurements. Multiphase units with pulse output (compatible with conventional data logging equipment) are also available. The major issue with current transformers, is that they are not shunt devices, so a breakout is still needed to monitor voltage, which since voltage may routinely drift by around $10 \%$, is crucial for accurate power measurement. Clearly, only one power logger which monitors voltage per phase would solve this, since once we have a log of voltage, plain current loggers could then be used for further disaggregation. Any tapping of voltage in, for example, explosive atmosphere environments, now has practical implications since if were to use current transformers, we may now have to package wiring. This means that current transformers effectively may not be always viewed as a non-invasive solution, but will still be required when high-frequency (sub 60 second) sampling of electrical feeds is needed. A solution may be found in a recent development in submetering, is a miniaturised non-fiscal sub meter, which can be fitted into a single DIN mounted fuseway. Indeed, submetering is now available which can take the form of an integrated sub meter and miniature circuit breaker, which can form a straight swap for an existing circuit breaker taking minutes, even between production runs. This combines the convenience of non-invasive instrumentation with the performance of a shunt device.

Clearly for monitoring energy delivery in the form of a fluid, be it water, air, or oil, or steam on natural gas, typically invasive metering is used. This can cause serious costs when production downtime may be in the order of thousands of euros per hour. Where submetering does exist, as with electricity and water, manual readings can be made, with the same restrictions. Some fiscal metering may find application, contact closure based gas meters usually operate by rotating a permanent magnet inside the meter housing, which magnetically couples with a reed switch attached to the outside of the case - these are known as R5 meters in the UK. . Consequently the most common type of meter is purely mechanical. An ultrasonic gas meter usually has two opposing transducers that both emit and receive ultrasonic signals. Transit-times are measured first in the direction of the flow and then opposite the flow. At low flow speed, relative to the speed of sound, the difference in transit time is directly proportional to the gas flow. Such a device was envisaged as long ago as
1994 [37]. Ultrasonic metering is non-invasive, but requires some planning; For example for metering of fluids such as natural gas, the pipe inner surface (roughness) may need to be a known quantity [38]. Similarly, ultrasonic metering offers an easy to fit solution for many other process gases and liquids including compressed air, steam, and water, and refrigerant. With metering strategy, a compromise is usually made between speed of installation, and cost effectiveness.

\section{DISCUSSION}

A basic data structure is proposed which offers the software engineer, the energy manager, and ultimately a standards body a 'way in' to constructing compatible energy datasets. Clearly an extensible structure such as MTConnect is required to add extra data where appropriate. The next step must be cooperation between all interested parties to achieve consensus on energy data for portability, functionality, and quality. While this paper looks at energy data from an operational and software engineering perspective, other analysts in the field may notice gaps or improvements in functionality, notably for meta data analysis, e.g. manufacturing data analysis which is based on small sample numbers when looking at vertical sectors [10]. Many analysis techniques commonplace in manufacturing are finding application in energy analysis [7], and additional data may be required to describe, for example, production schedules, and machine types. As exergy analysis becomes more popular too, data will deed to describe fuel types, embodied energy, and material throughput, as well as extra measurements from the manufacturing environment, such as chemical composition. Holistic audits will cover all material and energy flows from office supplies to manufactured items, all of which affect energy use, not least by effects on technical building services. As analysis techniques mature, we must be mindful that techniques such as AI, spectral analysis and complex event processing will place more exacting requirements on data quality and documentation. Finally and topically, manufacturers who increasingly see energy as a manufacturing process variable, will be looking to improve compatibility between manufacturing and energy data systems.

\section{CONCLUSIONS}

Energy data will find more use in manufacturing, to control processes as well as reduce costs. It is also clear that more sophisticated analysis techniques are becoming widespread. The idea that four main areas should be of importance when designing energy datasets has been stated, these being temporal, accuracy and precision of data, operational and energy supporting documentation. We have described some of the intricacies of a core dataset for building energy analysis, and some aspects of data which must be documented for enhanced functionality, not least to describe in more detail energy use patterns caused by manufacturing. A data design model has been presented as an example for a portable energy dataset. Trends in instrumentation are now making the performance division blurred between invasive and non invasive systems, meaning data gathering is possible with minimal impact on production. Improvements in data structure, reliability and quality and in instrumentation are vital if energy data is to be more effectively used as a process 
variable in manufacturing. The result will be greater efficiency, leading to reduced energy costs and greenhouse gas emissions.

\section{ACKNOWLEDGMENTS}

The authors would like to thank the European Commission and the partners of the European FP7 project "Knowledge, Awareness and Prediction of Man, Machine, Material and Method in Manufacturing" (www.kap-project.eu) for their support.

\section{REFERENCES}

[1] DECC UK Emissions Statistics. Department of Energy \& Climate Change, 3 Whitehall Place, London, SW1A 2AW, UK. http://www.decc.gov.uk/en/content/cms/statistics/climate stats/gg emiss ions/uk_emissions/uk_emissions.aspx Accesed March 2012.

[2] APERC, "Energy Efficiency Indicators. A Study Of Energy Efficiency Indicators For Industry In APEC Economies.," Asia Pacific Energy Research Centre, 2000.

[3] P. Stephenson and M. Paun, "Consumer advantages from half-hourly metering and load profiles in the UK competitive electricity market," Drpt2000: International Conference on Electric Utility Deregulation and Restructuring and Power Technologies, Proceedings, pp. 35-40 688, 2000.

[4] N. Brown, Wright, A.J, "DELIVERABLE 15. Report on Half-Hourly Metering Profiles " Institutute of Energy and Sustainable Development, DeMontfort University, Leicester, LE1 9BH, UK2008.

[5] N. Brown, et al., "Longitudinal analysis of energy metering data from non-domestic buildings," Building Research \& Information, vol. 38, pp. $80-91,2010$.

[6] B. Swords, et al., "An enterprise energy-information system," Applied Energy, vol. 85, pp. 61-69, 2008.

[7] N. Brown, Wright, A.J, "New Approaches to the Gathering, Analysis and Interpretation of Half Hourly Energy Metering Data from Buildings," International Journal of Low Carbon Technologies, vol. 2, 2007.

[8] W. Younger, "Using interval meter data for improved facility management," Energy Engineering: Journal of the Association of Energy Engineering, vol. 104, pp. 21-33, 2007.

[9] N. Brown, et al., "Novel Instrumentation for Monitoring After-Hours Consumption of Electrical Equipment," Energy and Buildings, 2011.

[10] R. Liddiard, "Characterising Space Use and Electricity Consumption in Non-domestic Buildings," PhD, Institute of Energy and Sustainable Development, DeMontfort University, Leicester, UK, 2012.

[11] G. Stuart, "Monitoring Energy Performance in Local Authority Buildings," PhD, IESD, DeMontfort University, Leicester, 2011.

[12] V. Ferrera, "The analysis of primary metered half-hourly electricity and gas consumption in municipal buildings " PhD, IESD, DeMontfort University, Leicester. Instituto Superior Técnico, Lisbon, 2009.

[13] G. Stuart, et al., "Rapid analysis of time series data to identify changes in electricity consumption patterns in UK secondary schools," Building and Environment, vol. 42, p. 1568, 2007.

[14] A. J. Wright, Brown, N., "The Analysis and interpretation of half hourly utility data in UK buildings.," presented at the Fourth International Conference Improving Energy Efficiency in Commercial Buildings, Frankfurt, 2008.

[15] Anon, "VDI 4602 Energy Management Standard: Energy Management. Terms, definitions," Verein Deutscher Ingenieure, Düsseldorf 2006 www.socialtext.net/data/workspaces/energy audit/attachments/timetable :20090323212237-0-20816/original/VDI\%25204602 en.pdf Accesed March 2012.

[16] ANSI/ MSE, "A Management System for Energy," ed, 2008. Georgia Institute of Technology, Enterprise Innovation Institute, Energy and Env. Mgmt. Ctr. 760 Spring Street NW, Atlanta, Georgia, USA.
[17] H. A. Chu Pulido and I. Sanchez Ramos, "Present conditions and perspectives of the standardization of the energy efficiency in Mexico; Actualidad y perspectivas del programa de normalizacion de la eficiencia energetica en Mexico," ed, 1999.

[18] N. Brown and A. Wright, "Non-Invasive and Cost Effective Monitoring of Energy Consumption Patterns for Electrical Equipment," 2008.

[19] B. Painter, et al., "Practical application of a sensor overlay system for building monitoring and commissioning," Energy and Buildings, 2012.

[20] P. Dekkers, "Complex Event Processing," ed: Radboud University Nijmegen, 2007. Nijmegen Institute for Computing and Information Sciences (NIII), Toernooiveld 16525 ED, Nijmegen, Netherlands.

[21] N. Brown, et al., "Machine vision in conjunction with a knowledgebased system for semi-automatic control of a gravure printing process," Proceedings of the Institution of Mechanical Engineers Part I-Journal of Systems and Control Engineering, vol. 218, pp. 583-593, 2004.

[22] L. Deng and E. Williams, "Measures and trends in energy use of semiconductor manufacturing," presented at the Electronics and the Environment, 2008. IEEE International Symposium on ISEE 2008., 2008.

[23] O. I. Avram and P. Xirouchakis, "Evaluating the use phase energy requirements of a machine tool system," Journal of Cleaner Production, vol. 19, pp. 699 - 711, 2011.

[24] ISO, "ISO 22029: Standard file format for spectral data exchange ", 2003. 1, ch. de la Voie-Creuse, CP 56, CH-1211 Geneva 20, Switzerland.

[25] BSI, "BS EN 1434-4:2007. Heat meters. Pattern approval tests.," ed, 2007. British Standards Institution, 389 Chiswick High Road London W4 4AL, UK

[26] BSI, "BS EN 60255-24:2001 IEC 60255-24:2001 Electrical relays Part 24: Common format for transient data exchange (COMTRADE) for power systems," ed, 2001.

[27] BSI, "DD CEN/TS 15231:2006 DRAFT FOR DEVELOPMENT Open data communication in building automation, controls and building management Mapping between Lonworks and BACnet," ed, 2006.

[28] BSI, "PD CEN/TR 16061:2010 Standards Application - Gas meters Smart Gas Meters," ed, 2010. British Standards Institution, 389 Chiswick High Road London W4 4AL, UK

[29] BSI, "BS ISO 12174:2003 Space data and information transfer systems. Telecommand. Architectural specification for the data management service," 2003.

[30] BSI, "Draft BS EN 62714-1 Engineering data exchange format for use in industrial automation systems engineering(AutomationML) - Part 1: Architecture and General Requirements," ed, 2012.

[31] CENELEC, "TECHNICAL REPORT CLC/TR 50403 Standardization and the liberalization of the energy market ", ed, 2002. 17, Avenue Marnix, B-1000 Brussels, Belgium.

[32] BSI, "BS EN 15232:2007 Energy performance of buildings. Impact of building automation, controls and building management," 2007.

[33] BSI, "BS EN 61970-405:2007 Energy management system application program interface (EMS-API) - Part 405: Generic eventing and Subscription (GES)," ed, 2007.

[34] E. Abele, Sielaff, T., Scniffer, A., Rothenbucher, S., "Analyzing Energy Consumption of Machine Tool Spindle Units and Identification of Potential for Improvements of Efficiency," Proceedings of the 18th CIRP International Conference on Life Cycle Engineering, Technische Universität Braunschweig, Braunschweig, Germany, , 2011.

[35] BSI, "EN 62053-61:1998 Electricity metering equipment (a.c.) Particular requirements," in EN 62053-61:1998, ed: BSI, 1999, p. 18.

[36] BSI, "Electricity metering. Glossary of terms PD IEC TR 62051:1999, IEC TR 62051:1999," ed: BSI, 2002, p. 60.

[37] T. J. Sheppard, "Solid-State Gas Metering - the Future," Flow Measurement and Instrumentation, vol. 5, pp. 103-106, Apr 1994.

[38] A. Calogirou, et al., "Effect of wall roughness changes on ultrasonic gas flowmeters," Flow Measurement and Instrumentation, vol. 12, pp. 219229, Jun 2001. 
